\title{
Online Appendix \\ Understanding Consumption Behavior: Evidence from Consumers' Reaction to Shopping Vouchers
}

\begin{abstract}
Kamhon Kan, Shin-Kun Peng, Ping Wang
Appendix A-Survey and Questionnaire Our survey used a multistage sampling scheme, where a subsample was selected from each region (a county or a municipality) in the first stage. There were in total 23 counties and municipalities. The number of individuals drawn from a region was proportional to its population size. Random subsamples for each region were drawn from the 2004-2005 Telephone Directory of the Chunghwa Telecom Inc., which is the sole provider of land phone services in Taiwan. Because some numbers were not listed and new numbers were not covered by this version of the telephone directory, to improve the representativeness of the set of telephone numbers drawn, for each number drawn from the electronic telephone directory, we substituted the last two digits with a random number. The second stage involved within-household sampling - that is, from all the individuals above 18 in a household we randomly selected one individual as an interviewee. For this purpose, we prepared a set of tables, and conditional on the number of male and female qualified individuals in a household, each table specified which individual was to be selected as an interviewee. The selection of which table was to be used for the within-household sampling was based on the last two digits of a household's telephone number. This is similar to the way that the Survey of Consumers selects a member in a household as respondent.
\end{abstract}

\section{Survey Questionnaire}

Selected Questions from the Survey Questionnaire

Q4. Did anyone in your household give you his/her shopping vouchers to you or use his/her vouchers jointly with yours?

(1) Yes (Go to Q4_1)

(2) No (Go to Q5)

(8) Refuse to answer (Go to Q5)

Q5. Was there anyone who does not live with you giving you his/her shopping vouchers to you or using his/her vouchers jointly with you?

(1) Yes, persons

(2) $\mathrm{No}$ 
(8) Refuse to answer

Q6. Did you use the shopping vouchers by yourself or you gave to other people?

(1) All at my own disposal

(2) Gave all to other people

(3) Kept some for own disposal and gave some to other people

(4) Kept some for own disposal and some for other uses

(5) Put them to other uses

Q7. What was the total value of the shopping vouchers that were at your disposal?
(01) $\$ 0$ (go to Q18)
(02) $\$ 1-500$
(03) $\$ 501-1000$
(04) $\$ 1001-1500$
(05) \$1501-2000
(06) $\$ 2001-2500$
(07) $\$ 2501-3000$
(08) $\$ 3001-3500$
(09) $\$ 3501-4000$
(10) $\$ 4001-4500$
(11) $\$ 4501-5000$
(12) $\$ 5001-5500$
(13) $\$ 5501-6000$
(14) $\$ 6001-6500$
(15) $\$ 6501-7000$
(16) $\$ 7001-7500$
(17) $\$ 7501-8000$
(18) $\$ 8001-8500$
(19) $\$ 8501-9000$
(20) $\$ 9001-9500$
(21) \$9501-10000
(22) \$10001-12000
(23) $\$ 12001-13000$
(24) $\$ 13001-14000$
(25) \$14001-15000
(26) $\$ 15001-16000$
(27) \$16001-17000
(28) \$17001-18000
(29) \$18001-19000
(31) 20001 and Over
(97) Don’t know
(30) \$19001-20000
(98) Refuse to answer

Q8. Did you use any of the shopping vouchers that were at your disposal?

(1) Yes, I used all of them (Please go to Q10)

(2) Yes, I used some of them

(3) No, I did not use any of them (Please go to Q18)

(7) Don't know

(8) Refuse to answer

Q9. How much was the amount of the shopping vouchers that you used?
(01) $\$ 1-500$
(02) $\$ 501-1000$
(03) \$1001-1500
(04) \$1501-2000
(05) $\$ 2001-2500$
(06) $\$ 2501-3000$
(07) \$3001-3500
(08) $\$ 3501-4000$
(09) \$4001-4500
(10) $\$ 4501-5000$
(11) $\$ 5001-5500$
(12) $\$ 5501-6000$
(13) \$6001-6500
(14) $\$ 6501-7000$
(15) $\$ 7001-7500$
(16) $\$ 7501-8000$
(17) $\$ 8001-8500$
(18) $\$ 8501-9000$
(19) $\$ 9001-9500$
(20) $\$ 9501-10000$
(21) \$10001-12000
(22) \$12001-13000
(23) \$13001-14000
(24) $\$ 14001-15000$
(25) \$15001-16000
(26) $\$ 16001-17000$
(27) \$17001-18000
(28) \$18001-19000
(29) \$19001-20000
(30) $\$ 20001$ or more
(97) Don't know
(98) Refuse to answer

Q10. What kinds of goods did you use shopping vouchers to pay for? 
(01) Food and drinks (i.e., groceries, alcohol and tobacco) (Answer Q12)

(02) Household necessities (e.g., personal care products, kitchen supplies and utensils) (Answer Q12)

(03) Consumer durables (e.g., consumer electronics, furniture, electric appliances, cookware, automobiles, bicycles) (Answer Q13)

(04) Apparel and accessories, jewelry, home decor (Answer Q13)

(05) Toys, books, stationery, magazines, CDs, and DVDs (Answer Q13)

(06) Services (meals at restaurants, barber shops, beauty salons, leisure travel, etc.) (Answer Q14)

(07) Medicine, health and fitness products (Go to Q15 after answering Q11)

(08) Tuition or tutoring fees (Go to Q15 after answering Q11)

(09) Transportation fares (i.e., taxi fares, train tickets) (Go to Q15 after answering Q11)

(10) Rent (i.e., rent for apartment or shop) (Go to Q15 after answering Q11)

(11) Donations (Go to Q15 after answering Q11)

(12) Given away as gifts (Go to Q15 after answering Q11)

(13) Sold to others (Go to Q15 after answering Q11)

(14) Other use (Go to Q15 after answering Q11)

(97) Don't know

(98) Refuse to answer

Q12. How much of the shopping vouchers at your disposal was spent on food and drinks or household necessities (items in (01) and (02) of Q10)?
(01) $\$ 500$ or Less
(02) $\$ 501-1000$
(03) $\$ 1001-1500$
(04) \$1501-2000
(05) \$2001-2500
(06) $\$ 2501-3000$
(07) $\$ 3001-3500$
(08) $\$ 3501-4000$
(09) $\$ 4001-4500$
(10) $\$ 4501-5000$
(11) $\$ 5001-5500$
(12) $\$ 5501-6000$
(13) $\$ 6001-6500$
(14) $\$ 6501-7000$
(15) $\$ 7001-7500$
(16) $\$ 7501-8000$
(17) $\$ 8001-8500$
(18) $\$ 8501-9000$
(19) $\$ 9001-9500$
(20) \$9501-10000
(21) \$10001-12000
(22) \$12001-13000
(23) \$13001-14000
(24) $\$ 14001-15000$
(25) \$15001-16000
(26) \$16001-17000
(27) \$17001-18000
(28) \$18001-19000
(29) \$19001-20000
(30) $\$ 20001$ or more
(97) Don't know
(98) Refuse to answer

Q12_1. What was the nature of those purchases?

(1) I would have made the purchases even without receiving shopping vouchers (Go to Q12_2)

(2) I made the purchases, only because of receiving shopping vouchers (Go to Q15)

(3) The purchases were made, because of discounts when using shopping vouchers (Go to Q15) 
(4) All of the above are true to some extent (Go to Q12_2)

(8) Refuse to answer (Go to Q15)

Q12_2. Were the items that you bought more or less expensive than, or almost the same price as, you originally planned?

(1) More expensive

(2) Less expensive

(3) Almost the same price

(6) Other

(7) Don’t know

(8) Refuse to answer

Q13. How much of the shopping vouchers at your disposal was spent on consumer durables, apparel and accessories, toys, books, stationery, magazines, CDs, and DVDs (items in (03)-(05) of Q10)?
(01) $\$ 500$ or Less
(02) \$501-1000
(03) \$1001-1500
(04) \$1501-2000
(05) \$2001-2500
(06) \$2501-3000
(07) \$3001-3500
(08) \$3501-4000
(09) \$4001-4500
(10) $\$ 4501-5000$
(11) $\$ 5001-5500$
(12) \$5501-6000
(13) $\$ 6001-6500$
(14) $\$ 6501-7000$
(15) $\$ 7001-7500$
(16) \$7501-8000
(17) \$8001-8500
(18) \$8501-9000
(19) \$9001-9500
(20) \$9501-10000
(21) \$10001-12000
(22) \$12001-13000
(23) \$13001-14000
(24) \$14001-15000
(25) \$15001-16000
(26) \$16001-17000
(27) \$17001-18000
(28) \$18001-19000
(29) \$19001-20000
(30) $\$ 20001$ or more
(97) Don't know
(98) Refuse to answer

Q13_1. What was the nature of these purchases?

(1) I would have made the purchases even without receiving shopping vouchers (Go to Q13_2)

(2) I made the purchases, only because of receiving shopping vouchers (Go to Q15)

(3) The purchases were made, because of discounts when using shopping vouchers (Go to Q15)

(4) All of the above are true to some extent (Go to Q13_2)

(8) Refuse to answer (Go to Q15)

Q13_2. Were the items that you bought more or less expensive than, or almost the same price as, you originally planned?

(1) More expensive

(2) Less expensive

(3) Almost the same price

(6) Other

(7) Don't know

(8) Refuse to answer 
Q14. How much of the shopping vouchers at your disposal was spent on meals at restaurants, barber shops, beauty salons, leisure travel, etc. (items in (06) of Q10)?
(01) $\$ 500$ or Less
(02) $\$ 501-1000$
(03) $\$ 1001-1500$
(04) $\$ 1501-2000$
(05) $\$ 2001-2500$
(06) $\$ 2501-3000$
(07) $\$ 3001-3500$
(08) $\$ 3501-4000$
(09) $\$ 4001-4500$
(10) $\$ 4501-5000$
(11) $\$ 5001-5500$
(12) \$5501-6000
(13) \$6001-6500
(14) $\$ 6501-7000$
(15) $\$ 7001-7500$
(16) $\$ 7501-8000$
(17) $\$ 8001-8500$
(18) \$8501-9000
(19) $\$ 9001-9500$
(20) $\$ 9501-10000$
(21) $\$ 10001-12000$
(22) \$12001-13000
(23) \$13001-14000
(24) \$14001-15000
(25) \$15001-16000
(26) \$16001-17000
(27) \$17001-18000
(28) \$18001-19000
(29) \$19001-20000
(30) $\$ 20001$ or more
(97) Don't know
(98) Refuse to answer

Q14_1. What was the nature of those purchases?

(1) I would have made the purchases even without receiving shopping vouchers (Go to Q14_2)

(2) I made the purchases, only because of receiving shopping vouchers (Go to Q15)

(3) The purchases were made, because of discounts when using shopping vouchers (Go to Q15)

(4) All of the above are true to some extent (Go to Q14_2)

(8) Refuse to answer (Go to Q15)

Q14_2. Were the items that you bought more or less expensive than, or almost the same price as, you originally planned?

(1) More expensive

(2) Less expensive

(3) Almost the same price

(6) Other

(7) Don't know

(8) Refuse to answer

Q15. When using shopping vouchers, did you use cash or credit cards, because the total purchase amount exceeded the value of your shopping vouchers?

(1) Yes

(2) No (Go to Q16)

(8) Refuse to answer (Go to Q16)

Q15_1. How much more did you spend? 
(01) $\$ 500$ or Less

(02) \$501-1000

(03) \$1001-1500

(04) \$1501-2000

(05) \$2001-2500

(06) \$2501-3000

(07) \$3001-3500

(08) \$3501-4000

(09) \$4001-4500

(10) $\$ 4501-5000$

(11) $\$ 5001-5500$

(12) $\$ 5501-6000$

(13) \$6001-6500

(14) $\$ 6501-7000$

(15) \$7001-7500

(16) $\$ 7501-8000$

(17) $\$ 8001-8500$

(18) $\$ 8501-9000$

(19) \$9001-9500

(20) \$9501-10000

(21) \$10001-12000

(22) \$12001-13000

(23) \$13001-14000

(24) \$14001-15000

(25) \$15001-16000

(26) \$16001-17000

(27) \$17001-18000

(28) \$18001-19000

(29) \$19001-20000

(30) $\$ 20001$ or more

(97) Don't know

(98) Refuse to answer

Q19_2. Do you agree with the following claim?

The shopping vouchers program has worsened Taiwan's fiscal situation

(1) Strongly agree

(2) Agree

(3) Somewhat agree

(4) Disagree

(5) Strongly disagree

(7) Don’t know

(8) Refuse to answer

Q21. Supposing the government hands out shopping vouchers worth $\$ 3,600$ per person in the second half of 2009 again, how will you use them if there is no discount for using shopping vouchers?

(1) Spend them on items that I would have purchased even without receiving shopping vouchers

(2) Spend them on items that I would not have purchased without receiving shopping vouchers

(3) Half and half

(4) Other (e.g., donations)

(7) Don't know

(8) Refuse to answer

Q22. Supposing the government decides to refund part of your 2008 personal income tax, and the amount is roughly $\$ 3,600$ per person (the same amount as the value of shopping vouchers received by your household) in the second half of 2009, how will you use the income tax refund?

(1) I will spend it on items that I would have purchased even without receiving the tax refund

(2) I will spend it on items that I would not have purchased without receiving the tax refund

(3) Savings or investment

(4) I will use some on shopping, and savings or investment

(5) Other (i.e., donations)

(7) Don’t know 
(8) Refuse to answer

Q32. Are you optimistic about Taiwan's economy next year (2010)?

(1) Very optimistic

(2) Somewhat optimistic

(3) Somewhat pessimistic

(4) Very pessimistic

(7) Don't know

(8) Refuse to answer 


\section{Appendix B-Responses to Hypothetical Questions}

In questions Q21 and Q22, we asked how the respondents would use their vouchers and tax refunds, respectively. See Table A8 for tabulations of the responses. In the hypothetical shopping vouchers question, we asked whether they would use them on (i) items that would have been purchased even without receiving shopping vouchers or (ii) on items that would not have been purchased without receiving shopping vouchers, where we allowed our respondents to answer (iii) "spending on both types of items" or (iv) other purposes. Likewise, in the hypothetical tax refunds question, we asked whether they would use the refund (i) on planned spending, (ii) savings or investment, (iii) use on unplanned spending, allowing them to answer (iv) "use some of it on shopping, and savings or investment" or (v) other purposes.

In response to the hypothetical question on a future voucher program, $67.4 \%$ of our respondents indicated that they would use the vouchers on planned spending, while only about $4.0 \%$ intended to use them on unplanned spending, $27.0 \%$ of them on a mix of planned and unplanned spending, and $1.7 \%$ of them for other purposes. We observe a similar pattern of responses to the hypothetical tax rebates question. While $67.8 \%$ of our respondents would use the tax rebates on

planned spending or savings/investment, $24.8 \%$ of them intended a mix of uses, while $1.8 \%$ of them would use the tax rebates for other purposes.

Assuming that respondents answering mixed use (i.e., (iii) in Q21 and answering (iv) in Q22) in the two hypothetical questions intended to spend half of their vouchers or tax rebates on planned items, then MPC estimates based on the hypothetical questions would be 0.175 and 0.18 under the future shopping vouchers and tax rebates senarius, respectively. 


\section{Appendix C-Aggregate Implications}

This Appendix checks the consistency of our estimation results and explores the implications of the voucher program on the aggregate consumption.

Panel (A)

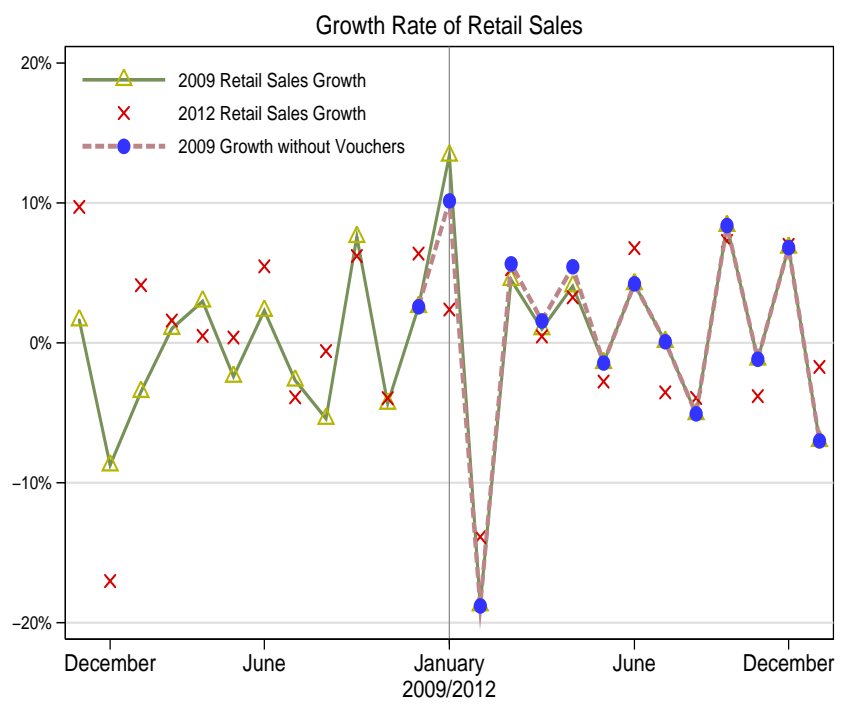

Panel (B)

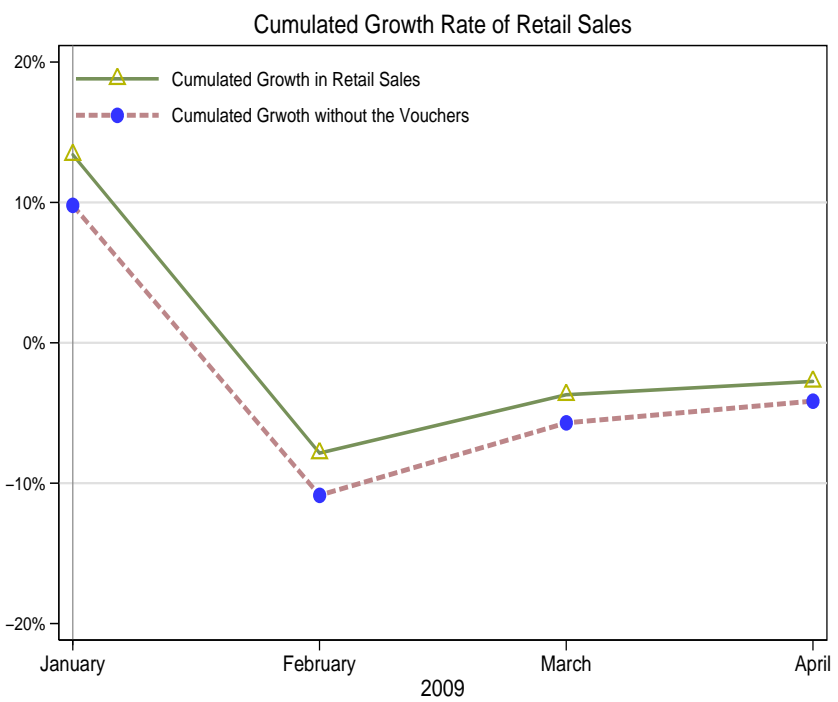

Figure A1: Counterfactual Analysis on Growth of Retail Sales Notes: Data on retail sales come from Department of Statistics, Ministry of Economic Affairs, which conducted monthly surveys of retailers and restaurants and imputes retail and restaurant sales for the whole country.

We check the consistency of our results by comparing the retail (inclusive of restaurants) sales growth without vouchers with the comparable growth data in 2012. To construct the figures without vouchers, we assume that shopping vouchers generated $34.12 \%$ (i.e., our MPC estimate inclusive of the effect of outof-pocket expenditure) of new spending and $36.00 \%$ of this new spending took place in January 2009, with $30 \%, 20 \%$ and $14 \%$ spending having occurred in the second to the fourth months (the same fraction of spending over time as found by Sahm et al., 2010. See their Table 4). We subtract the voucher-related new spending from the retail sales to compute the counterfactual month-over-month growth.

Panel (A) of Figure A1 plots the 2009 retail sales growth, the counterfactual growth rates assuming the absence of vouchers, and the 2012 growth rates. We use the 2012 sales growth as a benchmark, because in both 2009 and 2012 the Chinese New Year happened in the late February. The lunar new year affects the seasonality of consumption significantly. Moreover, the trend of quarterly real GDP growth for 2011 and 2012 was very similar to that for 2008-2009-both are U-shaped. ${ }^{\text {A1 }}$ Panel (A) of Figure A1 shows that the 2009 sales growth without

\footnotetext{
${ }^{\mathrm{A} 1}$ The real quarterly GDP growth rates for 2011 and 2012 are $7.63 \%, 4.76 \%, 3.63 \%, 1.18 \%, 0.53 \%, 0.08 \%$, $1.35 \%$ and $3.85 \%$, while those for $2008-2009$ are $7.55 \%, 5.66 \%,-1.23 \%,-7.53 \%,-8.12 \%,-6.58 \%,-1.41 \%$ and $8.82 \%$.
} 
vouchers follows a similar pattern as that of 2012. This implies our estimation based on survey data is consistent with the aggregate phenomenon.

We next explore the aggregate implications of Taiwan's voucher program by constructing a counterfactual cumulated retail growth measure. ${ }^{\text {A2 }}$ Panel (B) of Figure A1 plots the counterfactual cumulated retail sales growth in comparison with the actual one. It indicates that the program boosted the cumulated sales growth by $10.02 \%$ over the first four months after the launch of the program.

\footnotetext{
${ }^{\text {A2}}$ Define the cumulated retail growth rate as $\Delta_{t}^{R}=\left(R_{t}-R_{0}\right) / R_{0}$, where $R_{t}$ is the retail sales in month $t$ with $t=0$ denoting December 2008. The counterfactual one is defined as $\tilde{\Delta}_{t}^{R}=\left[\left(R_{t}-d_{t} \cdot \widehat{\mathrm{MPC}} \cdot V\right)-R_{0}\right] / R_{0}$, where $V$ is the total value of vouchers disbursed, $d_{t}$ is the fraction of spending in month $t$ as specified above, and $\widehat{M P C}$ is our estimate of MPC inclusive of out-of-pocket spending.
} 
Appendix D-Tables 


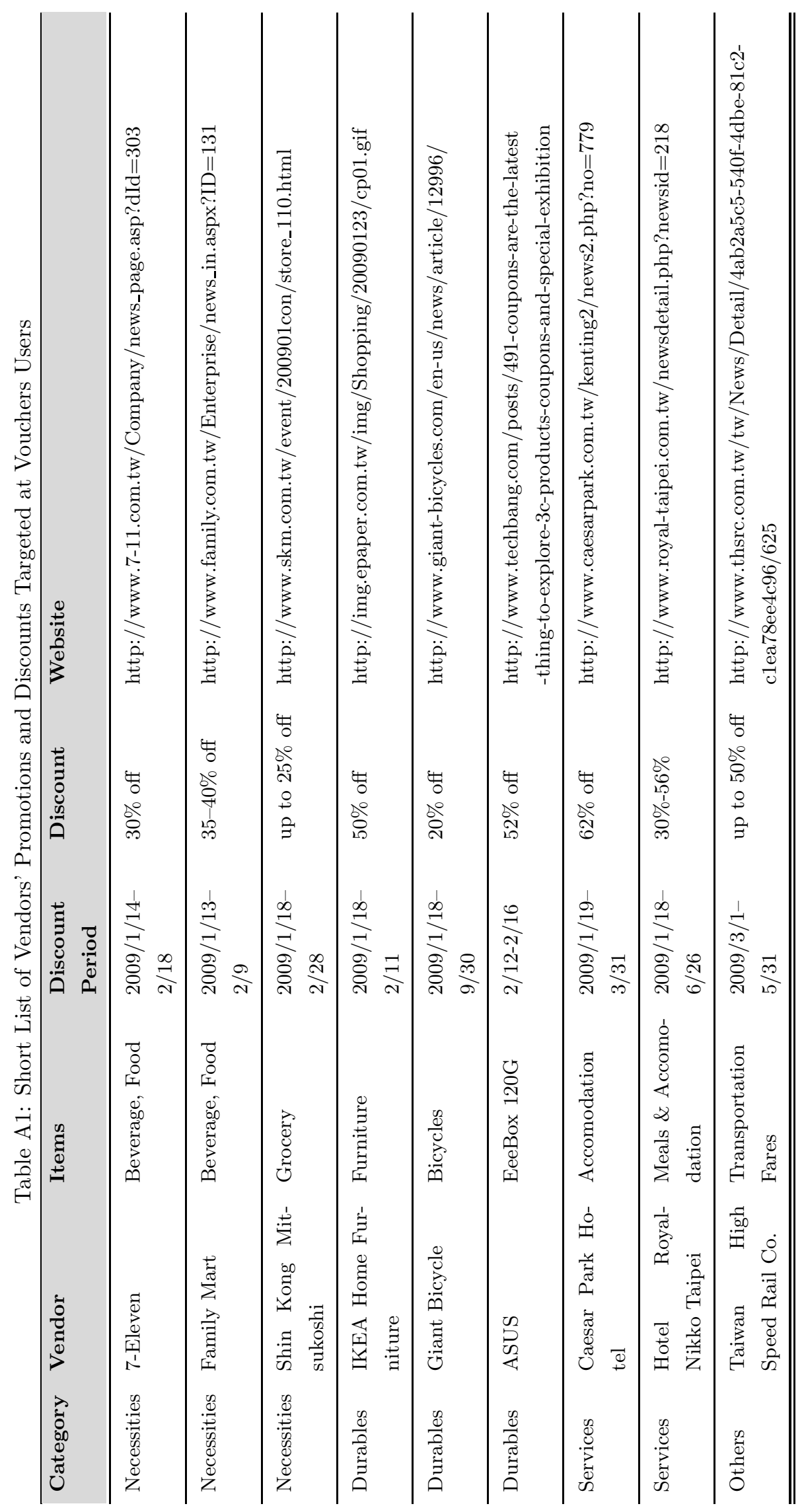


Table A2: Telephone Interview Outcomes

\begin{tabular}{|c|c|c|c|}
\hline \multicolumn{2}{|c|}{ Calls completed } & \multirow{2}{*}{$\begin{array}{r}12077 \\
3832\end{array}$} & \multirow{2}{*}{$\begin{array}{r}43.13 \% \\
13.7 \%\end{array}$} \\
\hline (1) & Interviews completed & & \\
\hline$(2)$ & Non-residential phone number & 1888 & $6.7 \%$ \\
\hline (3) & Under 18 or non-citizen & 97 & $0.4 \%$ \\
\hline (4) & Disabled & 197 & $0.7 \%$ \\
\hline$(5)$ & Do not speak Chinese or Hokkien dialect & 22 & $0.1 \%$ \\
\hline (6) & Have already been interviewed & 20 & $0.1 \%$ \\
\hline (7) & Within-household sampled respondent not at home & 1095 & $3.9 \%$ \\
\hline (8) & Refused & 4816 & $17.2 \%$ \\
\hline (9) & Redirected to mobile phone & 75 & $0.3 \%$ \\
\hline (10) & Did not claim shopping vouchers yet & 24 & $0.1 \%$ \\
\hline (11) & Interrupted during interview & 11 & $0.00 \%$ \\
\hline \multicolumn{2}{|c|}{ Calls failed } & 15922 & $43.1 \%$ \\
\hline (12) & Not answered & 11409 & $40.8 \%$ \\
\hline (13) & Fax machine & 1556 & $5.6 \%$ \\
\hline (14) & Line busy & 1101 & $3.9 \%$ \\
\hline (15) & Answering machine & 101 & $0.4 \%$ \\
\hline (16) & Number not in service & 8 & $0.0 \%$ \\
\hline (17) & Telephone out of order & 447 & $1.6 \%$ \\
\hline (18) & Number temporarily out of service & 15 & $0.1 \%$ \\
\hline (19) & Telephone set to "do not disturb" & 1285 & $4.6 \%$ \\
\hline \multicolumn{2}{|c|}{ CATI system crashed } & 1 & $0.0 \%$ \\
\hline \multicolumn{2}{|c|}{ Calls attempted } & 28000 & $100 \%$ \\
\hline
\end{tabular}


Table A3: Categories and Items of Voucher Spending*

\begin{tabular}{|c|c|c|c|c|}
\hline (1) & $(2)$ & (3) & $(4)$ & $(5)$ \\
\hline $\begin{array}{l}\text { "Whether } \\
\text { spent on } \\
\text { items" } \\
\text { Asked }\end{array}$ & Items incluced & Purchased & $\begin{array}{ll}\text { "Value spent on } \\
\text { the category" } \\
\text { Asked }\end{array}$ & Purchased \\
\hline$\langle 1\rangle$ & Groceries & $56.4 \%$ & $\langle A\rangle$ Necessities & $70.3 \%$ \\
\hline$\langle 2\rangle$ & $\begin{array}{l}\text { Household necessities (e.g., personal care } \\
\text { products, kitchen supplies and utensils) }\end{array}$ & $62.7 \%$ & & \\
\hline$\langle 3\rangle$ & $\begin{array}{l}\text { Consumer durables (e.g., consumer elec- } \\
\text { tronics, furniture, electrical appliances, } \\
\text { cookware, automobiles, bicycles) }\end{array}$ & $29.0 \%$ & $\langle B\rangle$ Durables & $57.7 \%$ \\
\hline$\langle 4\rangle$ & $\begin{array}{l}\text { Apparel and accessories, jewelry, home } \\
\text { decor }\end{array}$ & $33.2 \%$ & & \\
\hline$\langle 5\rangle$ & Toys, books, magazines, CDs, and DVDs & $9.7 \%$ & & \\
\hline$\langle 6\rangle$ & $\begin{array}{l}\text { Services (restaurants, barber shops, } \\
\text { beauty salons, traveling, etc.) }\end{array}$ & $23.0 \%$ & $\langle C\rangle$ Services & $23.0 \%$ \\
\hline$\langle 7\rangle$ & Medicine, health and fitness products & $4.2 \%$ & $\langle D\rangle$ Others & $16.0 \%$ \\
\hline$\langle 8\rangle$ & Tuition for private lessons & $2.3 \%$ & & \\
\hline$\langle 9\rangle$ & Transportation fares & $9.5 \%$ & & \\
\hline$\langle 10\rangle$ & Rent & $0.03 \%$ & & \\
\hline$\langle 11\rangle$ & Donations & $1.5 \%$ & & \\
\hline$\langle 12\rangle$ & As gifts to other people & $1.7 \%$ & & \\
\hline$\langle 13\rangle$ & Sold for cash to somebody else & $0.5 \%$ & & \\
\hline$\langle 14\rangle$ & Other uses & $1.0 \%$ & & \\
\hline
\end{tabular}

Notes: The 14 categories in column (1) are aggregated into 4 categories in column (4). In columns (3) and (5), the numbers are for the percentage of respondents having made a purchase of items in the category. The value of voucher spending was not asked for category $\langle D\rangle$. 
Table A4: Patterns of Shopping Vouchers on Hand and Used

\begin{tabular}{|c|c|c|c|c|c|c|}
\hline \multirow[b]{2}{*}{ Amount } & \multicolumn{6}{|c|}{ Frequency [Percentage] } \\
\hline & On (1) & Used & $\begin{array}{c}\text { (3) } \\
\text { Necessities } \\
\end{array}$ & Durables & $\begin{array}{c}(5) \\
\text { Services } \\
\end{array}$ & Out-of-Pocket \\
\hline$\$ 0$ & $\begin{array}{c}0 \\
{[0 \%]}\end{array}$ & $\begin{array}{c}122 \\
{[3.7 \%]}\end{array}$ & $\begin{array}{c}865 \\
{[29.7 \%]}\end{array}$ & $\begin{array}{l}1232 \\
{[42.3 \%]}\end{array}$ & $\begin{array}{l}2240 \\
{[77.0 \%]}\end{array}$ & $\begin{array}{c}430 \\
{[16.36 \%]}\end{array}$ \\
\hline$\$ 1-500$ & {$\left[\begin{array}{l}3 \\
0.1 \%\end{array}\right]$} & {$[0.4 \%]$} & {$\left[\begin{array}{c}65 \\
2.2 \%\end{array}\right]$} & {$[2.5 \%]$} & {$\left[\begin{array}{c}65 \\
2.2 \%]\end{array}\right.$} & {$\left[\begin{array}{c}572 \\
21.76 \%\end{array}\right]$} \\
\hline$\$ 501-1000$ & {$[0.3 \%]$} & {$[0.8 \%]$} & {$\left[\begin{array}{l}105 \\
3.6 \%]\end{array}\right.$} & {$\left[\begin{array}{l}125 \\
4.3 \%\end{array}\right]$} & {$[3.4 \%]$} & {$\left[\begin{array}{r}405 \\
15.41 \%\end{array}\right]$} \\
\hline$\$ 1001-1500$ & {$[0.1 \%]$} & {$\left[\begin{array}{c}22 \\
0.7 \%\end{array}\right]$} & {$\left[\begin{array}{c}87 \\
3.0 \%]\end{array}\right]$} & $\begin{array}{l}116 \\
{[4.0 \%]}\end{array}$ & {$\left[\begin{array}{c}78 \\
{[2.7 \%]}\end{array}\right]$} & $\begin{array}{c}186 \\
{[7.07 \%]}\end{array}$ \\
\hline$\$ 1501-2000$ & {$\left[\begin{array}{l}24 \\
{[0.7 \%]}\end{array}\right.$} & $\left.\begin{array}{l}49 \\
{[1.5 \%]}\end{array}\right]$ & $\begin{array}{l}146 \\
5.0 \%]\end{array}$ & $\begin{array}{l}142 \\
{[4.9 \%]}\end{array}$ & $\left.\begin{array}{c}74 \\
{[2.5 \%]}\end{array}\right]$ & {$\left[\begin{array}{c}190 \\
7.23 \%]\end{array}\right.$} \\
\hline$\$ 2001-2500$ & {$[0.2 \%]$} & {$\left[\begin{array}{c}32 \\
1.0 \%\end{array}\right]$} & $\left.\begin{array}{l}112 \\
3.9 \%\end{array}\right]$ & $\begin{array}{c}99 \\
{[3.4 \%]}\end{array}$ & {$\left[\begin{array}{c}58 \\
{[2.0 \%]}\end{array}\right.$} & [ $3.80 \%]$ \\
\hline$\$ 2501-3000$ & {$[0.2 \%]$} & {$\left[\begin{array}{c}39 \\
{[1.2 \%]}\end{array}\right.$} & {$\left[\begin{array}{l}115 \\
4.0 \%]\end{array}\right.$} & $\begin{array}{l}118 \\
4.1 \%]\end{array}$ & {$\left[\begin{array}{c}49 \\
{[1.7 \%]}\end{array}\right.$} & {$[5.06 \%]$} \\
\hline$\$ 3001-3500$ & {$[0.0 \%]$} & {$\left[\begin{array}{c}35 \\
{[1.1 \%]}\end{array}\right.$} & {$\left[\begin{array}{r}73 \\
{[2.5 \%]}\end{array}\right.$} & {$\left[\begin{array}{l}65 \\
{[2.2 \%]}\end{array}\right.$} & {$\left[\begin{array}{c}34 \\
{[1.2 \%]}\end{array}\right]$} & {$\left[\begin{array}{c}68 \\
2.59 \%\end{array}\right]$} \\
\hline$\$ 3501-4000$ & {$\left[\begin{array}{l}1324 \\
39.6 \%]\end{array}\right.$} & {$\left[\begin{array}{l}1,142 \\
34.5 \%]\end{array}\right.$} & {$\left[\begin{array}{c}467 \\
{[16.1 \%]}\end{array}\right.$} & $\begin{array}{c}312 \\
{[10.7 \%]}\end{array}$ & {$\left[\begin{array}{c}93 \\
3.2 \%]\end{array}\right.$} & {$[2.70 \%]$} \\
\hline$\$ 4001-4500$ & {$[0.3 \%]$} & {$\left[\begin{array}{c}23 \\
0.7 \%\end{array}\right]$} & $\begin{array}{c}50 \\
{[1.7 \%]}\end{array}$ & {$\left[\begin{array}{c}30 \\
{[1.0 \%]}\end{array}\right]$} & {$\left[\begin{array}{l}11 \\
0.4 \%\end{array}\right]$} & {$[1.07 \%]$} \\
\hline$\$ 4501-5000$ & {$\left[\begin{array}{c}21 \\
0.6 \%]\end{array}\right.$} & {$\left[\begin{array}{c}42 \\
{[1.3 \%}\end{array}\right]$} & {$\left[\begin{array}{c}62 \\
2.1 \%]\end{array}\right]$} & {$\left[\begin{array}{l}55 \\
1.9 \%\end{array}\right]$} & $\left.\begin{array}{c}17 \\
{[0.6 \%}\end{array}\right]$ & {$[3.04 \%]$} \\
\hline$\$ 5001-5500$ & {$\left[\begin{array}{l}10 \\
0.3 \%\end{array}\right]$} & $\begin{array}{c}16 \\
{[0.5 \%]}\end{array}$ & {$\left[\begin{array}{l}55 \\
1.9 \%\end{array}\right]$} & $\begin{array}{c}38 \\
{[1.3 \%]}\end{array}$ & {$[0.2 \%]$} & {$\left[\begin{array}{c}22 \\
0.84 \%\end{array}\right]$} \\
\hline$\$ 5501-6000$ & {$[0.3 \%]$} & {$[0.7 \%]$} & {$\left[\begin{array}{c}59 \\
2.0 \%]\end{array}\right.$} & {$\left[\begin{array}{c}42 \\
{[1.4 \%]}\end{array}\right.$} & {$\left[\begin{array}{l}12 \\
0.4 \%]\end{array}\right.$} & {$\left[\begin{array}{c}52 \\
1.98 \%\end{array}\right]$} \\
\hline$\$ 6001-6500$ & {$[0.1 \%]$} & {$\left[\begin{array}{c}10 \\
0.3 \%]\end{array}\right.$} & {$\left[\begin{array}{l}26 \\
0.9 \%]\end{array}\right.$} & {$\left[\begin{array}{c}16 \\
0.6 \%]\end{array}\right.$} & {$[0.1 \%]$} & {$[0.53 \%]$} \\
\hline$\$ 6501-7000$ & {$\left[\begin{array}{c}5 \\
5 \\
0.2 \%\end{array}\right]$} & {$\left[\begin{array}{c}25 \\
{[0.8 \%}\end{array}\right]$} & {$[1.6 \%]$} & {$\left[\begin{array}{c}32 \\
1.1 \%\end{array}\right]$} & {$[0.1 \%]$} & {$\left[\begin{array}{c}15 \\
0.57 \%\end{array}\right]$} \\
\hline$\$ 7001-7500$ & {$\left[\begin{array}{c}450 \\
13.5 \%]\end{array}\right.$} & $\left.\begin{array}{c}395 \\
{[11.9 \%}\end{array}\right]$ & {$\left[\begin{array}{l}150 \\
5.2 \%\end{array}\right]$} & $\left.\begin{array}{l}87 \\
{[3.0 \%]}\end{array}\right]$ & {$\left[\begin{array}{c}22 \\
0.8 \%\end{array}\right]$} & {$[0.53 \%]$} \\
\hline$\$ 7501-8000$ & {$\left[\begin{array}{c}6 \\
0.2 \%\end{array}\right]$} & {$\left[\begin{array}{c}18 \\
0.5 \%\end{array}\right.$} & {$\left[\begin{array}{c}41 \\
1.4 \%\end{array}\right]$} & {$\left[\begin{array}{c}22 \\
0.8 \%\end{array}\right]$} & {$\left[\begin{array}{c}6 \\
0.2 \%\end{array}\right]$} & {$[0.65 \%]$} \\
\hline$\$ 8001-8500$ & {$\left[\begin{array}{c}7 \\
0.2 \%\end{array}\right]$} & $\begin{array}{c}12 \\
{[0.4 \%]}\end{array}$ & {$\left[\begin{array}{c}18 \\
0.6 \%\end{array}\right.$} & $\begin{array}{c}13 \\
{[0.5 \%]}\end{array}$ & {$\left[\begin{array}{c}4 \\
0.1 \%]\end{array}\right]$} & {$\left[\begin{array}{c}5 \\
0.19 \%\end{array}\right]$} \\
\hline$\$ 8501-9000$ & {$[0.1 \%]$} & {$\left[\begin{array}{l}25 \\
0.8 \%\end{array}\right]$} & {$[1.3 \%]$} & {$[0.6 \%]$} & {$[0 \%]$} & {$\left[\begin{array}{c}8 \\
0.30 \%\end{array}\right]$} \\
\hline$\$ 9001-9500$ & {$\left[\begin{array}{c}6 \\
0.2 \%\end{array}\right]$} & {$\left[\begin{array}{c}9 \\
0.3 \%\end{array}\right]$} & {$\left[\begin{array}{c}13 \\
0.5 \%]\end{array}\right]$} & {$\left[\begin{array}{c}4 \\
{[0.1 \%]}\end{array}\right]$} & {$\left[\begin{array}{c}2 \\
0.1 \%]\end{array}\right]$} & {$\left[\begin{array}{c}4 \\
{[0.15 \%]}\end{array}\right.$} \\
\hline$\$ 9501-10000$ & {$[0.2 \%]$} & {$[0.8 \%]$} & {$\left[\begin{array}{l}50 \\
1.7 \%\end{array}\right]$} & {$\left[\begin{array}{c}32 \\
1.1 \%]\end{array}\right.$} & {$[0.2 \%]$} & {$[0.95 \%]$} \\
\hline$\$ 10001-12000$ & {$\left[\begin{array}{c}439 \\
{\left[\begin{array}{c}13.1 \% \\
\end{array}\right]}\end{array}\right.$} & $\begin{array}{c}472 \\
{[14.3 \%]}\end{array}$ & {$\left[\begin{array}{c}94 \\
3.2 \%\end{array}\right]$} & {$\left[\begin{array}{l}86 \\
{[3.0 \%]}\end{array}\right.$} & {$\left[\begin{array}{c}15 \\
{[0.5 \%]}\end{array}\right]$} & {$[1.71 \%]$} \\
\hline$\$ 12001-13000$ & {$\left[\begin{array}{c}7 \\
0.2 \%]\end{array}\right]$} & {$\left[\begin{array}{c}5 \\
0.2 \%\end{array}\right]$} & {$\left[\begin{array}{c}24 \\
0.8 \%\end{array}\right]$} & {$\left[\begin{array}{c}11 \\
0.4 \%\end{array}\right]$} & {$[0.0 \%]$} & {$\left[\begin{array}{c}9 \\
0.34 \%\end{array}\right]$} \\
\hline$\$ 13001-14000$ & {$[0.1 \%]$} & {$\left[\begin{array}{l}3 \\
0.1 \%\end{array}\right]$} & {$\left[\begin{array}{c}16 \\
0.6 \%\end{array}\right.$} & {$\left[\begin{array}{c}11 \\
0.4 \%]\end{array}\right.$} & {$[0.1 \%]$} & {$\left[\begin{array}{c}5 \\
0.19 \%\end{array}\right]$} \\
\hline$\$ 14001-15000$ & {$\left[\begin{array}{c}542 \\
16.2 \%\end{array}\right]$} & $\begin{array}{c}399 \\
{[12.1 \%]}\end{array}$ & {$\left[\begin{array}{c}62 \\
2.1 \%\end{array}\right]$} & $\begin{array}{c}66 \\
{[2.3 \%]}\end{array}$ & {$\left[\begin{array}{c}6 \\
0.2 \%\end{array}\right]$} & {$\left[\begin{array}{l}31 \\
1.18 \%]\end{array}\right.$} \\
\hline$\$ 15001-16000$ & {$[0.1 \%]$} & [ $0.1 \%$ ] & {$\left[\begin{array}{l}10 \\
0.3 \%\end{array}\right]$} & {$[0.2 \%]$} & {$[0 \%]$} & {$[0.38 \%]$} \\
\hline$\$ 16001-17000$ & {$[0.3 \%]$} & {$[0.1 \%]$} & {$[0.2 \%]$} & {$[0.3 \%]$} & {$[0 \%]$} & {$[0.31 \%]$} \\
\hline$\$ 17001-18000$ & {$\left[\begin{array}{l}247 \\
{[7.4 \%]}\end{array}\right.$} & $\begin{array}{l}176 \\
{[5.3 \%]}\end{array}$ & {$\left[\begin{array}{c}28 \\
1.0 \%\end{array}\right]$} & {$\left[\begin{array}{l}30 \\
1.0 \%\end{array}\right]$} & {$[0.0 \%]$} & {$[0.5 \%$} \\
\hline$\$ 18001-19000$ & $\begin{array}{c}0 \\
{[0 \%}\end{array}$ & $\left.\begin{array}{c}0 \\
0 \%\end{array}\right]$ & {$\left[\begin{array}{l}3 \\
0.1 \%\end{array}\right]$} & {$\left[\begin{array}{c}0 \% \\
0 \%]\end{array}\right.$} & $\begin{array}{c}0 \% \\
{[0 \%]}\end{array}$ & {$\left[\begin{array}{c}1 \\
0.04 \%\end{array}\right]$} \\
\hline$\$ 19001-20000$ & {$\left[\begin{array}{c}1 \\
0.0 \%\end{array}\right]$} & {$\left[\begin{array}{c}1 \\
0.0 \%]\end{array}\right]$} & {$\left[\begin{array}{c}5 \\
0.2 \%\end{array}\right]$} & {$[0 \%]$} & {$[0.0 \%]$} & {$[0.19 \%]$} \\
\hline$\$ 20001$ and above & $\begin{array}{l}177 \\
{[5.3 \%]}\end{array}$ & $\begin{array}{c}141 \\
{[4.3 \%]}\end{array}$ & {$\left[\begin{array}{c}18 \\
{[0.6 \%]}\end{array}\right.$} & {$\left[\begin{array}{c}27 \\
{[0.9 \%]}\end{array}\right.$} & {$[0.0 \%]$} & [ $2.89 \%]$ \\
\hline Sample Average & $\begin{array}{c}8886.68 \\
(5463.97) \\
\end{array}$ & $\begin{array}{c}7920.78 \\
(5381.53) \\
\end{array}$ & $\begin{array}{c}3725.95 \\
(4242.88) \\
\end{array}$ & $\begin{array}{c}2882.91 \\
(4193.51) \\
\end{array}$ & $\begin{array}{c}680.16 \\
(1857.76) \\
\end{array}$ & $\begin{array}{c}2613.0947 \\
(4334.9947) \\
\end{array}$ \\
\hline Total & 3345 & 3309 & 2910 & 2910 & 2910 & 2629 \\
\hline
\end{tabular}

Notes: In column (1), among the 3,832 respondents, 487 indicated that they had given or sold all vouchers to others and were excluded. In column (2), among the 3,345 respondents, 36 answered "don't know" to the amount of their voucher spending (Q8 or Q9) and were excluded. In columns (3)-(5), among the 3309 respondents, 399 answered "don't know" to the questions on the amount of their voucher spending (Q12_1, Q13_1 \& Q14_1) or items they have spent on (Q10) were excluded. In column (6), among the 2910 respondents, 281 respondents answered "don't know" to the question on the amount of their out-of-pocket spending. The sample averages (standard deviation in parentheses) are computed using the midpoints of the ranges. 
Table A5: Marginal Propensity to Consume: Corrected for Reporting and Recall Errors

\begin{tabular}{|c|c|c|}
\hline & $\begin{array}{c}(1) \\
M P C\end{array}$ & $\begin{array}{l}\quad \quad(2) \\
\quad M P C \\
\text { (Excluding } \\
\text { Effects of } \\
\text { Discounts) }\end{array}$ \\
\hline Estimate & $\begin{array}{c}0.247 \\
(0.007)\end{array}$ & $\begin{array}{c}0.167 \\
(0.006)\end{array}$ \\
\hline Observations & 2797 & 2797 \\
\hline \multicolumn{3}{|c|}{$\begin{array}{l}\text { Notes: The following corrections are made. (i) Use } \\
\text { the sum of the reported voucher spending as the total } \\
\text { voucher spending for respondents who reported having } \\
\text { used no vouchers on other items (category }\langle D\rangle \text { ). (ii) } \\
\text { Drop the observation when a respondent reported hav- } \\
\text { ing spent on category }\langle D\rangle \text { (others) and the sum of the } \\
\text { estimates of voucher spending on necessities, durables } \\
\text { and service is greater than the estimate of total voucher } \\
\text { spending. Standard errors in parentheses. }\end{array}$} \\
\hline
\end{tabular}


Table A6: Multivariate Analysis of Spending Shares

\begin{tabular}{|c|c|c|c|c|c|c|}
\hline \multirow{3}{*}{ Age $\leq 29$} & \multirow{2}{*}{\multicolumn{2}{|c|}{$\begin{array}{l}\text { Necessities } \\
(1)\end{array}$}} & \multirow{2}{*}{\multicolumn{2}{|c|}{ 3) Durables }} & \multicolumn{2}{|c|}{ Services } \\
\hline & & & & & (5) & $(6)$ \\
\hline & $\begin{array}{c}0.010 \\
(0.265)\end{array}$ & $\begin{array}{c}0.013 \\
(0.264)\end{array}$ & $\begin{array}{c}0.6376^{* *} \\
(0.2732)\end{array}$ & $\begin{array}{c}0.6544^{* *} \\
(0.2730)\end{array}$ & $\begin{array}{c}0.3356 \\
(0.3305)\end{array}$ & $\begin{array}{c}0.3856 \\
(0.3327)\end{array}$ \\
\hline Age 30 & $\begin{array}{c}0.261 \\
(0.245)\end{array}$ & $\begin{array}{l}0.258 \\
(0.245)\end{array}$ & $\begin{array}{c}0.5289^{* *} \\
(0.2606)\end{array}$ & $\begin{array}{c}0.5247^{* *} \\
(0.2601)\end{array}$ & $\begin{array}{c}0.3544 \\
(0.3207)\end{array}$ & $\begin{array}{c}0.3534 \\
(0.3218)\end{array}$ \\
\hline Age 40 & $\begin{array}{c}0.070 \\
(0.230)\end{array}$ & $\begin{array}{c}0.061 \\
(0.230)\end{array}$ & $\begin{array}{c}0.2795 \\
(0.2447)\end{array}$ & $\begin{array}{c}0.2577 \\
(0.2445)\end{array}$ & $\begin{array}{l}-0.0993 \\
(0.3087)\end{array}$ & $\begin{array}{c}-0.1031 \\
(0.3100)\end{array}$ \\
\hline Age 50 & $\begin{array}{c}0.033 \\
(0.203)\end{array}$ & $\begin{array}{l}0.028 \\
(0.203)\end{array}$ & $\begin{array}{c}0.1906 \\
(0.2217)\end{array}$ & $\begin{array}{c}0.1781 \\
(0.2213)\end{array}$ & $\begin{array}{l}-0.1631 \\
(0.2894)\end{array}$ & $\begin{array}{l}-0.1722 \\
(0.2901)\end{array}$ \\
\hline Elementary school & $\begin{array}{c}0.051 \\
(0.187)\end{array}$ & $\begin{array}{c}0.054 \\
(0.187)\end{array}$ & $\begin{array}{c}-0.6323^{* * *} \\
(0.2115)\end{array}$ & $\begin{array}{c}-0.6163^{* * *} \\
(0.2123)\end{array}$ & $\begin{array}{c}-0.9105^{* * *} * \\
(0.3045)\end{array}$ & $\begin{array}{c}-0.8858^{* * *} \\
(0.3062)\end{array}$ \\
\hline College & $\begin{array}{c}0.123 \\
(0.163)\end{array}$ & $\begin{array}{c}0.128 \\
(0.163)\end{array}$ & $\begin{array}{c}0.3616^{* *} \\
(0.1656)\end{array}$ & $\begin{array}{c}0.3806^{* *} \\
(0.1653)\end{array}$ & $\begin{array}{c}0.5124 * * * \\
(0.1841)\end{array}$ & $\begin{array}{c}0.5190 * * * \\
(0.1837)\end{array}$ \\
\hline Married & $\begin{array}{c}0.147 \\
(0.158)\end{array}$ & $\begin{array}{c}0.123 \\
(0.162)\end{array}$ & $\begin{array}{l}-0.0480 \\
(0.1659)\end{array}$ & $\begin{array}{l}-0.1291 \\
(0.1696)\end{array}$ & $\begin{array}{c}0.1983 \\
(0.2094)\end{array}$ & $\begin{array}{c}0.0643 \\
(0.2127)\end{array}$ \\
\hline Kids & $\begin{array}{c}0.016 \\
(0.160)\end{array}$ & $\begin{array}{l}-0.013 \\
(0.160)\end{array}$ & $\begin{array}{l}-0.0006 \\
(0.1688)\end{array}$ & $\begin{array}{l}-0.0882 \\
(0.1696)\end{array}$ & $\begin{array}{l}-0.1577 \\
(0.2073)\end{array}$ & $\begin{array}{l}-0.2869 \\
(0.2071)\end{array}$ \\
\hline Female & $\begin{array}{c}0.345^{* * * *} \\
(0.127)\end{array}$ & $\begin{array}{c}0.336^{* * * *} \\
(0.129)\end{array}$ & $\begin{array}{l}0.2527^{*} \\
(0.1317)\end{array}$ & $\begin{array}{l}0.2226^{*} \\
(0.1331)\end{array}$ & $\begin{array}{c}0.3529 * * \\
(0.1563)\end{array}$ & $\begin{array}{l}0.2895^{*} \\
(0.1576)\end{array}$ \\
\hline Family income-Missing & $\begin{array}{c}-0.354^{* *} \\
(0.176)\end{array}$ & $\begin{array}{r}-0.342^{*} \\
(0.176)\end{array}$ & $\begin{array}{l}-0.2339 \\
(0.1822)\end{array}$ & $\begin{array}{l}-0.2038 \\
(0.1830)\end{array}$ & $\begin{array}{l}-0.0297 \\
(0.2191)\end{array}$ & $\begin{array}{c}0.0129 \\
(0.2194)\end{array}$ \\
\hline Family income-Zero & $\begin{array}{l}-0.079 \\
(0.188)\end{array}$ & $\begin{array}{l}-0.079 \\
(0.187)\end{array}$ & $\begin{array}{l}-0.2075 \\
(0.1959)\end{array}$ & $\begin{array}{l}-0.2109 \\
(0.1955)\end{array}$ & $\begin{array}{l}-0.3266 \\
(0.2353)\end{array}$ & $\begin{array}{c}-0.3322 \\
(0.2346)\end{array}$ \\
\hline $\begin{array}{l}\text { Family income } \\
1_{\text {St }} \text { Quartile }\end{array}$ & $\begin{array}{l}-0.152 \\
(0.226)\end{array}$ & $\begin{array}{l}-0.147 \\
(0.226)\end{array}$ & $\begin{array}{l}-0.2164 \\
(0.2378)\end{array}$ & $\begin{array}{l}-0.2056 \\
(0.2382)\end{array}$ & $\begin{array}{c}-0.5984^{* *} \\
(0.2976)\end{array}$ & $\begin{array}{c}-0.5937^{* *} \\
(0.2977)\end{array}$ \\
\hline $\begin{array}{l}\text { Family income } \\
4^{\text {th }} \text { Quartile }\end{array}$ & $\begin{array}{c}-0.548^{* *} \\
(0.220)\end{array}$ & $\begin{array}{c}-0.548^{* *} \\
(0.221)\end{array}$ & $\begin{array}{l}-0.1562 \\
(0.2279)\end{array}$ & $\begin{array}{l}-0.1514 \\
(0.2285)\end{array}$ & $\begin{array}{c}0.0585 \\
(0.2669)\end{array}$ & $\begin{array}{c}0.0529 \\
(0.2673)\end{array}$ \\
\hline Income increased & $\begin{array}{l}-0.196 \\
(0.150)\end{array}$ & $\begin{array}{l}-0.199 \\
(0.150)\end{array}$ & $\begin{array}{l}-0.1603 \\
(0.1556)\end{array}$ & $\begin{array}{l}-0.1718 \\
(0.1559)\end{array}$ & $\begin{array}{l}-0.2849 \\
(0.1892)\end{array}$ & $\begin{array}{l}-0.2992 \\
(0.1897)\end{array}$ \\
\hline $\begin{array}{l}\text { Vouchers on hand } \\
\text { - Below NT } \$ 3,500\end{array}$ & & $\begin{array}{l}-0.059 \\
(0.134)\end{array}$ & & $\begin{array}{l}-0.2150 \\
(0.1407)\end{array}$ & & $\begin{array}{c}-0.5154^{* * *} \\
(0.1751)\end{array}$ \\
\hline $\begin{array}{l}\text { Vouchers on hand } \\
\text { - Above NT } \$ 10,001\end{array}$ & & $\begin{array}{c}0.159 \\
(0.182)\end{array}$ & & $\begin{array}{c}0.3835^{* *} \\
(0.1906)\end{array}$ & & $\begin{array}{l}0.0851 \\
(0.2195)\end{array}$ \\
\hline Economy-optimistic & $\begin{array}{l}-0.061 \\
(0.196)\end{array}$ & $\begin{array}{l}-0.069 \\
(0.195)\end{array}$ & $\begin{array}{l}-0.0841 \\
(0.2039)\end{array}$ & $\begin{array}{l}-0.0983 \\
(0.2036)\end{array}$ & $\begin{array}{l}-0.1704 \\
(0.2465)\end{array}$ & $\begin{array}{l}-0.1766 \\
(0.2455)\end{array}$ \\
\hline Economy-pessimistic & $\begin{array}{l}-0.098 \\
(0.162)\end{array}$ & $\begin{array}{l}-0.091 \\
(0.162)\end{array}$ & $\begin{array}{l}-0.1622 \\
(0.1698)\end{array}$ & $\begin{array}{l}-0.1412 \\
(0.1706)\end{array}$ & $\begin{array}{l}-0.2596 \\
(0.2115)\end{array}$ & $\begin{array}{l}-0.2221 \\
(0.2122)\end{array}$ \\
\hline Economy-DK & $\begin{array}{l}0.040 \\
(0.221)\end{array}$ & $\begin{array}{c}0.048 \\
(0.221)\end{array}$ & $\begin{array}{l}-0.2612 \\
(0.2424)\end{array}$ & $\begin{array}{l}-0.2341 \\
(0.2430)\end{array}$ & $\begin{array}{l}-0.4608 \\
(0.3149)\end{array}$ & $\begin{array}{l}-0.3902 \\
(0.3160)\end{array}$ \\
\hline Constant & $\begin{array}{c}1.607 * * * \\
(0.257)\end{array}$ & $\begin{array}{c}1.650^{* * *} \\
(0.272)\end{array}$ & $\begin{array}{l}1.2599 * * * \\
(0.2747)\end{array}$ & $\begin{array}{c}1.4104^{* * *} \\
(0.2893)\end{array}$ & $\begin{array}{l}-0.0086 \\
(0.3431)\end{array}$ & $\begin{array}{l}0.3528 \\
(0.3561)\end{array}$ \\
\hline \# respondents & & & & 2910 & & \\
\hline
\end{tabular}

Note: Standard errors in parentheses.

$*, * *, * * *$ denote statistically significant at the $10 \%, 5 \%$, and $1 \%$ levels, respectively. 
Table A7: Multivariate Analysis of MPC

\begin{tabular}{|c|c|c|c|c|}
\hline & \multicolumn{2}{|c|}{$M P C$} & \multicolumn{2}{|c|}{$\begin{array}{l}\text { Out-of-pocket } \\
\text { spending }\end{array}$} \\
\hline & (1) & $(2)$ & $(3)$ & $(4)$ \\
\hline Age $\leq 29$ & $\begin{array}{c}0.146^{* * * *} \\
(0.032)\end{array}$ & $\begin{array}{c}0.146^{* * *} \\
(0.032)\end{array}$ & $\begin{array}{c}-1.10 \mathrm{e}+03^{* * *} \\
(369.076)\end{array}$ & $\begin{array}{c}-1.01 \mathrm{e}+03^{* * *} \\
(365.037)\end{array}$ \\
\hline Age 30 & $\begin{array}{l}0.053^{*} \\
(0.031)\end{array}$ & $\begin{array}{l}0.052^{*} \\
(0.031)\end{array}$ & $\begin{array}{l}-433.653 \\
(353.791)\end{array}$ & $\begin{array}{l}-450.779 \\
(349.326)\end{array}$ \\
\hline Age 40 & $\begin{array}{c}0.017 \\
(0.029)\end{array}$ & $\begin{array}{l}0.010 \\
(0.029)\end{array}$ & $\begin{array}{l}-171.215 \\
(338.047)\end{array}$ & $\begin{array}{l}-234.455 \\
(334.145)\end{array}$ \\
\hline Age 50 & $\begin{array}{c}0.002 \\
(0.026)\end{array}$ & $\begin{array}{c}0.002 \\
(0.026)\end{array}$ & $\begin{array}{l}-360.735 \\
(309.079)\end{array}$ & $\begin{array}{l}-382.490 \\
(305.216)\end{array}$ \\
\hline Elementary school & $\begin{array}{l}0.001 \\
(0.025)\end{array}$ & $\begin{array}{c}0.002 \\
(0.025)\end{array}$ & $\begin{array}{c}-849.490 * * * \\
(301.021)\end{array}$ & $\begin{array}{c}-745.466^{* *} \\
(297.503)\end{array}$ \\
\hline College & $\begin{array}{c}0.005 \\
(0.019)\end{array}$ & $\begin{array}{c}0.007 \\
(0.019)\end{array}$ & $\begin{array}{c}41.415 \\
(209.797)\end{array}$ & $\begin{array}{c}112.787 \\
(207.577)\end{array}$ \\
\hline Married & $\begin{array}{c}0.028 \\
(0.020)\end{array}$ & $\begin{array}{c}0.023 \\
(0.020)\end{array}$ & $\begin{array}{l}180.052 \\
(231.151)\end{array}$ & $\begin{array}{l}-183.682 \\
(232.714)\end{array}$ \\
\hline Kids & $\begin{array}{c}0.019 \\
(0.020)\end{array}$ & $\begin{array}{c}0.012 \\
(0.020)\end{array}$ & $\begin{array}{c}301.605 \\
(227.871)\end{array}$ & $\begin{array}{l}-81.653 \\
(230.148)\end{array}$ \\
\hline Female & $\begin{array}{l}-0.020 \\
(0.015)\end{array}$ & $\begin{array}{l}-0.021 \\
(0.015)\end{array}$ & $\begin{array}{l}-269.488 \\
(174.999)\end{array}$ & $\begin{array}{c}-422.807^{* *} \\
(174.586)\end{array}$ \\
\hline Family income-Missing & $\begin{array}{l}-0.032 \\
(0.023)\end{array}$ & $\begin{array}{l}-0.031 \\
(0.023)\end{array}$ & $\begin{array}{c}173.764 \\
(258.452)\end{array}$ & $\begin{array}{c}294.629 \\
(255.688)\end{array}$ \\
\hline Family income-Zero & $\begin{array}{l}-0.010 \\
(0.023)\end{array}$ & $\begin{array}{l}-0.011 \\
(0.023)\end{array}$ & $\begin{array}{l}-187.194 \\
(263.575)\end{array}$ & $\begin{array}{l}-205.153 \\
(260.247)\end{array}$ \\
\hline $\begin{array}{l}\text { Family income } \\
1^{\text {st }} \text { Quartile }\end{array}$ & $\begin{array}{l}-0.012 \\
(0.028)\end{array}$ & $\begin{array}{l}-0.012 \\
(0.028)\end{array}$ & $\begin{array}{l}-118.133 \\
(322.735)\end{array}$ & $\begin{array}{l}-64.438 \\
(318.725)\end{array}$ \\
\hline $\begin{array}{l}\text { Family income } \\
4^{\text {th }} \text { Quartile }\end{array}$ & $\begin{array}{l}-0.021 \\
(0.028)\end{array}$ & $\begin{array}{l}-0.020 \\
(0.028)\end{array}$ & $\begin{array}{c}829.249 * * * \\
(319.542)\end{array}$ & $\begin{array}{c}846.201^{* * *} \\
(315.556)\end{array}$ \\
\hline Income increased & $\begin{array}{c}0.006 \\
(0.019)\end{array}$ & $\begin{array}{c}0.005 \\
(0.019)\end{array}$ & $\begin{array}{c}163.122 \\
(219.552)\end{array}$ & $\begin{array}{c}104.252 \\
(216.886)\end{array}$ \\
\hline $\begin{array}{l}\text { Vouchers on hand } \\
\text { - Below NT } \$ 3,500\end{array}$ & & $\begin{array}{l}-0.009 \\
(0.017)\end{array}$ & & $\begin{array}{c}-1.15 \mathrm{e}+03^{* * *} \\
(194.651)\end{array}$ \\
\hline $\begin{array}{l}\text { Vouchers on hand } \\
\text { - Above NT } \$ 16,001\end{array}$ & & $\begin{array}{c}0.029 \\
(0.024)\end{array}$ & & $\begin{array}{c}1163.268^{* * *} \\
(271.758)\end{array}$ \\
\hline Economy-optimistic & $\begin{array}{c}0.013 \\
(0.022)\end{array}$ & $\begin{array}{c}0.013 \\
(0.024)\end{array}$ & $\begin{array}{c}642.646^{* *} \\
(272.349)\end{array}$ & $\begin{array}{c}579.074^{* *} \\
(269.071)\end{array}$ \\
\hline Economy-pessimistic & $\begin{array}{c}-0.035^{*} \\
(0.019)\end{array}$ & $\begin{array}{l}-0.034 \\
(0.021)\end{array}$ & $\begin{array}{l}-115.290 \\
(240.931)\end{array}$ & $\begin{array}{l}-20.335 \\
(238.166)\end{array}$ \\
\hline Economy-DK & $\begin{array}{c}0.006 \\
(0.025)\end{array}$ & $\begin{array}{c}0.009 \\
(0.027)\end{array}$ & $\begin{array}{l}-327.555 \\
(344.303)\end{array}$ & $\begin{array}{l}-129.950 \\
(341.276)\end{array}$ \\
\hline Constant & $\begin{array}{c}0.205^{* * * *} \\
(0.033) \\
\end{array}$ & $\begin{array}{c}0.210^{* * *} * \\
(0.036)\end{array}$ & $\begin{array}{c}2833.126^{* * *} \\
(385.609)\end{array}$ & $\begin{array}{c}3631.368^{* * *} \\
(405.578) \\
\end{array}$ \\
\hline $\begin{array}{l}R^{2} \\
\# \text { respondents }\end{array}$ & $\begin{array}{l}0.016 \\
2910\end{array}$ & $\begin{array}{l}0.017 \\
2910\end{array}$ & $\begin{array}{l}0.029 \\
2629\end{array}$ & $\begin{array}{l}0.055 \\
2629\end{array}$ \\
\hline
\end{tabular}

Notes: Standard errors in parentheses.

$*, * *, * * *$ denote statistically significant at the $10 \%, 5 \%$, and $1 \%$ levels, respectively. 
Table A8: Hypothetical Questions

Q21. If the government were to have a second voucher program, how would you use your shopping vouchers from the second shopping voucher program?

\section{Percentage}

(2)

(i) Spend on items that I would have purchased 67.4 even without receiving shopping vouchers

(ii) Spend on items that I would not have pur- 4.0 chased without receiving shopping vouchers

(iii) Spend on each category of items 27.0 (iv) Other (i.e., donations)

1.7

$67.4+13.5=80.9$

$4.0+13.5=17.5$
0 1.7

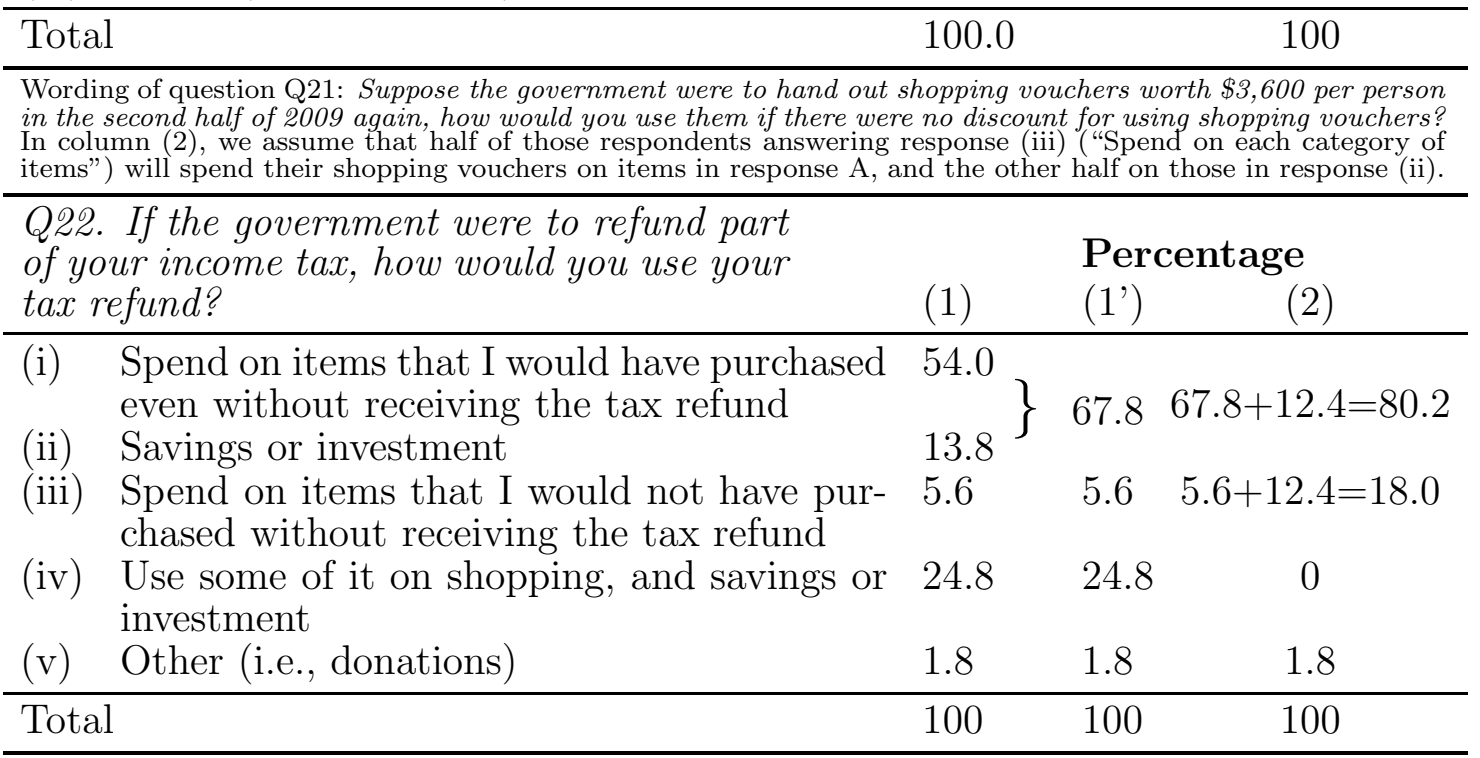

Notes: Wording of question Q22: Suppose the government decides to refund your 2008 personal income tax, and the amount is roughly $\$ 3600$ per person (the same amount as the value of the shopping vouchers received by your household) in the second half of 2009, how would you use the income tax refund?

In column (2), we assume that half of those respondents answering response (iv) ("Use some of it on shopping, and savings or investment") will spend their shopping vouchers on items in responses (i) and (ii), and the other half on those in response (iii). 